C-A/AP/514

MAP-doc-4378

February 2014

\title{
Details and justifications for the MAP concept specification for acceleration above $63 \mathrm{GeV}$
}

\author{
J.S. Berg
}

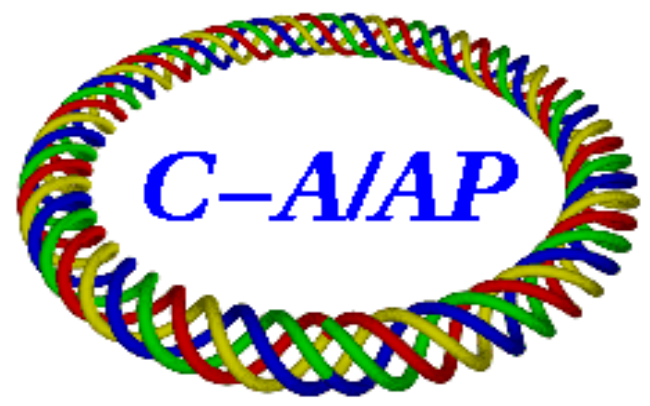

Collider-Accelerator Department Brookhaven National Laboratory

Upton, NY 11973

Notice: This document has been authorized by employees of Brookhaven Science Associates, LLC under Contract No. DE-AC02-98CH10886 with the U.S. Department of Energy. The United States Government retains a nonexclusive, paid-up, irrevocable, world-wide license to publish or reproduce the published form of this document, or allow others to do so, for United States Government purposes. 


\title{
Details and Justifications for the MAP Concept Specification for Acceleration Above 63 GeV
}

\author{
J. Scott Berg* \\ Brookhaven National Laboratory; Building 901A; PO Box 5000; Upton, NY 11973-5000; USA ${ }^{\dagger}$
}

(Dated: February 28, 2014)

\begin{abstract}
The Muon Accelerator Program (MAP) requires a concept specification for each of the accelerator systems. This document provides much of the information for the concept specification for the accelerators that bring the beam from a total energy of $63 \mathrm{GeV}$ to the maximum energy that will fit on the Fermilab site. Justifications and supporting references are included, providing more detail than will appear in the concept specification itself.
\end{abstract}

TABLE I. Interface parameters, performance requirements, and parameter constraints. Emittance growth can be defined as either $\Delta \epsilon / \epsilon$, where $\epsilon$ is the RMS emittance, or the maximum value of $|\Delta J| / J$ at $J=9 \epsilon / 2$, where $J$ is the action ( $\epsilon$ is the average of $J$ over the distribution).

\begin{tabular}{lrrr}
\hline \hline Input total energy $(\mathrm{GeV})$ & 63 & 750 & 1500 \\
Output total energy $(\mathrm{GeV})$ & 750 & 1500 & \\
Maximum circumference $(\mathrm{km})$ & & & 15 \\
\hline Normalized transverse emittance $(\mu \mathrm{m})$ & \multicolumn{2}{c}{25} & \\
Normalized longitudinal emittance $(\mathrm{mm})$ & \multicolumn{4}{c}{70} \\
Muons per sign & \multicolumn{3}{c}{$2 \times 10^{12}$} \\
Repetition rate $(\mathrm{Hz})$ & 12 & 6 \\
\hline Minimum average acceleration rate $(\mathrm{MeV} / \mathrm{m})$ & 3.5 & - \\
Maximum transverse emittance growth $(\%)$ & 6 & 3 & 3 \\
Maximum longitudinal emittance growth $(\%)$ & 6 & 3 & 3 \\
Minimum amplitude transmitted $(\sigma)$ & \multicolumn{4}{c}{4.5} & \\
\hline \hline
\end{tabular}

TABLE II. Loss when truncating a Gaussian distribution in sixdimensional phase space on an ellipsoid at a given multiple of the RMS size.

\begin{tabular}{lrrrr}
\hline \hline Truncation $\sigma$ & 3.0 & 3.5 & 4.0 & 4.5 \\
Loss $(\%)$ & 17 & 5.7 & 1.4 & 0.25 \\
\hline \hline
\end{tabular}

Table I specifies the requirements on the acceleration systems. The input and output energies are based on the machine energies defined in [1], as are the charge, emittances, and repetition rate. The maximum energy will be determined by the maximum machine circumference that will fit on the Fermilab site; I examined a site map and estimated this to be $15 \mathrm{~km}$, though it may be possible to make it slightly larger. The decay allowance (or equivalently the average acceleration rate) is not specified for that final stage, but decays will be taken into account when choosing that maximum energy. The average acceleration rate for the earlier stages is based on a transmission of $69 \%$ from 0.4 to $1500 \mathrm{GeV}$ kinetic energy proposed

\footnotetext{
* jsberg@bnl.gov; http://pubweb.bnl.gov/ jsberg/

$\dagger$ This manuscript has been authored by employees of Brookhaven Science Associates, LLC under Contract No. DE-AC02-98CH10886 with the U.S. Department of Energy. The United States Government retains a nonexclusive, paid-up, irrevocable, world-wide license to publish or reproduce the published form of this manuscript, or allow others to do so, for United States Government purposes.
}

TABLE III. Acceleration stages. Magnet fields increase approximately in proportion to beam momentum in a synchrotron. A hybrid synchrotron approximates this using interleaved fixed field magnets and bipolar ramped magnets.

\begin{tabular}{lrrrr}
\hline \hline Input total energy $(\mathrm{GeV})$ & 63 & $\approx 375$ & 750 & 1500 \\
Output total energy $(\mathrm{GeV})$ & $\approx 375$ & 750 & 1500 & $\approx 3000$ \\
Type & Synchrotron & $\begin{array}{r}\text { Hybrid } \\
\text { Synchrotron }\end{array}$ & $\begin{array}{r}\text { Hybrid } \\
\text { Synchrotron }\end{array}$ & $\begin{array}{r}\text { Hybrid } \\
\text { Synchrotron }\end{array}$ \\
\hline \hline
\end{tabular}

in [2]. These values are chosen consistently with [3]. Longitudinal emittance growth is based on the energy acceptance of the collider ring [4] in relation to the RMS energy spread and bunch length for a $70 \mathrm{~mm}$ longitudinal emittance, assuming we want to keep $3 \sigma$, and approximately distributing the allowed growth through the acceleration chain. I assume the same relative emittance growth is allowed transversely. The minimum amplitude transmitted is based on the assumption that a six-dimensional distribution in phase space is truncated on an ellipsoid at some multiple $n$ of the RMS size in each direction (the same multiple in each direction). The fractional loss from that truncation for a Gaussian disstribution would be

$$
\left(1+\frac{n^{2}}{2}+\frac{n^{4}}{8}\right) e^{-n^{2} / 2}
$$

The losses for some values of $n$ are tabulated in Table II. $n=4$ would keep losses well enough below losses from decays, but would leave little overhead for other effects, such as non-Gaussian distribution tails, nonlinearities, unexpected lattice errors, etc. I have therefore chosen $n=4.5$ to give a bit of extra overhead. If it is easier to create overhead in either the longitudinal or transverse direction, it may be more attracative to have different relative truncation amplitudes in the transverse and longitudinal planes.

Table III lists the proposed accelerator types to be used to accelerate above $63 \mathrm{GeV}$. Machine choices are based on the discussion in [5]. FFAGs do not appear to be cost-effective due to the large longitudinal emittance. Hybrid synchrotrons are attractive over all energy ranges, but it is not clear if field strength and quality can be achieved for the short pulse times required in the low energy stages. For the lowest energy stage, a dogbone recirculating linear accelerator is a possible alternative, but the ability to share a tunnel and magnet costs appear to favor the synchrotron option. Note the resemblance of the first two stages to [6].

The lattices will maintain a constant time of flight and tune through the acceleration process. The acceleration rate will be 
TABLE IV. Magnet parameters.

\begin{tabular}{lr}
\hline \hline Maximum fixed dipole field (T) & 10 \\
Maximum ramped dipole field (T) & 1.5 \\
Maximum ramped quadrupole field at beam (T) & 0.7 \\
Maximum ramped sextupole field at beam (T) & 0.5 \\
Maximum kicker field (T) & 0.2 \\
Maximum septum field (T) & 1.0 \\
Inter-magnet spacing (m) & 0.5 \\
\hline \hline
\end{tabular}

constant. Sextupoles will be included to correct chromaticity while canceling their lowest-order resonant driving terms. The energy breakpoint between the first two stages will be chosen to give the first two stages the same circumference. The highest possible RF frequency, up to $1300 \mathrm{MHz}$, will be used due to cost-effectiveness.

Magnet parameters are listed in Table IV. The maximum dipole field is the same that is assumed for the collider ring design [4], and is consistent with recent results for $\mathrm{Nb}_{3} \mathrm{Sn}$ dipoles [7]. The maximum ramped fields are what can be obtained for a dipole with a $3 \%$ silicon steel pole [8]. Higher fields could be obtained with grain-oriented steel or vanadium permendur. The former could have issues with field quality due to the pinning of field lines, and cannot be simulated with existing codes [9]. The latter would likely present a radiation hazard due to its significant cobalt content. A grain-oriented steel magnet has been built and tested, and reached $1.8 \mathrm{~T}$ with a $1.4 \mathrm{kHz}$ sinusoidal oscaillation [10]; we therefore believe that the pulsing rates required are achievable. Maximum quadrupole fields are estimated using Fig. 13 of [11], assuming a maximum field on the pole of $1.5 \mathrm{~T}$ (as for our dipole), a field quality of $0.1 \%$, and the beam occupying $70 \%$ of the magnet aperture. The corresponding maximum sextupole field is chosen by assuming the same pole tip field $(1.0 \mathrm{~T})$ and fraction of the magnet aperture as the quadrupole. I do not expect to interleave fixed-field quadrupoles or sextupoles because the quadrupoles and sextupoles are expected to occupy a small fraction of the circumference, and the inter-magnet spacing required would likely negate the advantage of increasing the average quadrupole gradient. Drift space between magnets is a working estimate [12].

Fields for injection and extraction magnets are specified primarily for the purpose of allocating sufficient space for those systems. A septum field is particularly difficult to choose, but [13] describes a built design that has a high field with low stray fields, and I base my choice on that design. For kickers, Nakamura [14, 15] describes designs that achieve fields as high as $0.3 \mathrm{~T}$ with rise times more rapid than we need. I've chosen $0.2 \mathrm{~T}$ to keep within the region where these designs have fields that are linear in current an in recognition that these fields are beyond what is conventionally used.

RF cavity parameters are given in Table V. $650 \mathrm{MHz}$ values are based on the SPL design [16]. The input power is based on values already obtained at $704 \mathrm{MHz}$ [16]. $1300 \mathrm{MHz}$ values are based on values achieved for ILC cavities [17]. Cells per cavity at $325 \mathrm{MHz}$ is based on the LEP2 cavities [18].

TABLE V. RF cavity parameters.

\begin{tabular}{lrrrr}
\hline \hline Frequency (MHz) & 325 & 650 & 975 & 1300 \\
\hline Gradient (MV/m) & 20 & 25 & 30 & 35 \\
Maximum energy gain per cell (MeV) & 9.22 & 5.77 & 4.61 & 4.04 \\
Maximum cells per cavity & 4 & 5 & 7 & 9 \\
Additional length at each end (cells) & & 1.5 & \\
Maximum input power per cavity (MW) & \multicolumn{5}{c}{1.2} \\
\hline \hline
\end{tabular}

R\&D improvements could result in more cost-effective acceleration. The ability to use higher-field fixed or ramped magnets would reduce circumferences and improve efficiency. Studies of fast ramp times in thin iron laminates could open up the possibility of using hybrid designs at lower energies and help us understand field and power loss limitations. A good shielding solution could allow the use of vanadium permendur. Improvements in modeling code could enable the use of grain-oriented steel. A cooling solution resulting in lower longitudinal emittance would reduce costs and could allow the use of more efficient FFAG designs at lower energies. A larger decay allowance would result in more efficient use of RF and longer pulse times for ramped magnets. Finally, it may be worthwhile to re-consider if RF frequencies above $1300 \mathrm{MHz}$ could be more cost-effective.
[1] J.-P. Delahaye et al., Enabling Intensity and Energy Frontier Science with a Muon Accelerator Facility in the U.S.: A White Paper Submitted to the 2013 U. S. Community Summer Study of the Division of Particles and Fields of the American Physical Society, Tech. Rep. FERMILAB-CONF-13-307-APC (Fermilab, 2014) arXiv:1308.0494 [physics.acc-ph].

[2] R. B. Palmer, "Draft muon collider parameters," MAP-doc-4318 (2011), http://map-docdb.fnal.gov.

[3] Jean-Pierre Delahaye, Robert B. Palmer, Thomas J. Roberts, Robert Ryne, Michael J. Syphers, and Katsuya Yonehara, "Criteria for comparing and assessing 6-D cooling channels," (2014), Muon Accelerator Program internal document, version dated Feb. 10, 2014.

[4] Y. Alexahin and E. Gianfelice-Wendt, "A 3-TeV muon collider lattice design," in Proceedings of IPAC2012, New Orleans, Louisiana, USA (IEEE, 2012) pp. 1254-1256.

[5] J. Scott Berg, FFAG Designs for Muon Collider Acceleration, informal report BNL-103716-2014-IR (Brookhaven National Laboratory, Upton, NY, 2014) also MAP-doc-4375, http: //map-docdb. fnal.gov/, BNL Collider-Accelerator Department Technical Note C-A/AP/498.

[6] D. J. Summers, L. M. Cremaldi, R. Godang, B. R. Kipapa, H. E. Rice, and R. B. Palmer, "Muon acceleration to $750 \mathrm{GeV}$ in the Tevatron tunnel for a $1.5 \mathrm{TeV} \mu^{+} \mu^{-}$collider," in Proceedings 
of PAC07, Albuquerque, New Mexico, USA (IEEE, 2007) pp. 3178-3180.

[7] M. Marchevsky et al., "Test of the high-field $\mathrm{Nb}_{3}$ Sn dipole magnet HD3b," IEEE Trans. Appl. Supercond. 24, 4002106 (2014).

[8] H. Witte, J. S. Berg, P. Kovach, M. D. Anerella, and M. L. Lopes, "Rapid cycling dipole magnet," in Proceedings of PAC2013, Pasadena, CA USA (2013) pp. 762-764.

[9] Holger Witte, FEA Simulations of Magnets with Grain Oriented Steel, informal report BNL-98381-2012-IR (Brookhaven National Laboratory, Upton, NY, 2012) also MAP-doc-4346, http://map-docdb.fnal.gov/.

[10] D. J. Summers, L. M. Cremaldi, T. L. Hart, L. P. Perera, M. Reep, H. Witte, S. Hansen, M. L. Lopes, and J. Reidy, "Test of a 1.8 Tesla, $400 \mathrm{~Hz}$ dipole for a muon synchrotron," in Proceedings of IPAC2012, New Orleans, Louisiana, USA (IEEE, 2012) pp. 3542-3544.

[11] Jack Tanabe, Iron Dominated Electromagnets: Design, Fabrication, Assembly and Measurements, report SLAC-R-754 (Stanford Linear Accelerator Center, Stanford, CA, 2005).

[12] H. Witte, verbal discussion (2011).

[13] G. H. Schröder, W. Weisser, E. Carlier, J. H. Dieperink, L. Ducimetière, B. Goddard, C. Lazar, M. Mayer, E. B. Vossen- berg, and W. Weterings, "A novel eddy current septum magnet for SPS extraction towards LHC and CNGS," in Proceedings of EPAC 2000, Vienna, Austria (2000) pp. 2370-2372.

[14] Eiji Nakamura, "Fast-rise high-field kicker magnet operating in saturation," Nucl. Instrum. Methods A 612, 50-55 (2009).

[15] Eiji Nakamura, "Fast-rise high-field multi-turn coil kicker magnet operating in saturation region," Nucl. Instrum. Methods A 618, 22-29 (2010).

[16] J. Plouin, G. Devanz, and S. Chel, "Optimized RF design of 704 $\mathrm{MHz}$ beta=1 cavity for pulsed proton drivers," in Proceedings of SRF2011, Chicago, IL, USA (2011) pp. 157-161.

[17] Rong-Li Geng, "SRF technical status and future R\&D," presentation at LCWS12: International Workshop on Future Linear Colliders, University of Texas at Arlington, USA, 22-26 October 2012 (2012).

[18] Daniel Boussard, "Operational experience with the LEP2 SC cavity system," in Fifth European Particle Accelerator Conference, edited by S. Myers, A. Pacheco, R. Pascual, Ch. Petit-JeanGenaz, and J. Poole (Institute of Physics Publishing, Bristol, UK, 1996) pp. 187-191. 